\title{
Análisis del entrenamiento resistido en el perfil glicémico: una revisión bibliográfica
}

\author{
Analysis of the resistance training in the glycemic profile: a bibliographic review \\ *,**Gaspar Pinto da Silva, ***,****Claudio Hernández-Mosqueira, \\ $* * * * *$ Gustavo Pavéz-Adasme, *Sandro Fernandes da Silva.
}

Pinto, G., Hernández, C., Pavéz. \& Fernandes, S. (2017). Análisis del entrenamiento resistido en el perfil glicémico: una revisión bibliográfica. Revista Ciencias de la Actividad Física UCM, Nº 18(2) julio-diciembre, 1-8.

\section{RESUMEN}

La presente revisión bibliográfica surge de la necesidad de revisar la evidencia sobre el efecto del entrenamiento resistido en el perfil glicémico. La búsqueda de información se realiza en las bases de datos Ebsco, PubMed, Google Académico y Researchgate, restringida a publicaciones de los años 2011 a 2016. Se utilizaron las siguientes palabras clave: Entrenamiento de Resistencia y de control de la diabetes, el Entrenamiento de Resistencia y el control de glucémico, el Entrenamiento de Resistencia y Control de la Salud. Fueron encontrados 841 artículos, a continuación, se excluyeron los artículos que no cumplían con los criterios de inclusión del estudio, de estos 75 fueron excluidos porque no manifestaran el efecto del entrenamiento de resistencia en la glucosa. Finalmente solo 6 estudios fueron seleccionados y analizados para la presente investigación. Los resultados de esta revisión muestran que los métodos de ER, se consideran importantes componentes cuando el objetivo principal es preservar el buen estado de salud, interviniendo positivamente en el perfil glucémico y minimizando los factores de riesgo inherentes a una dieta pobre y estilo de vida sedentario, demostrando que no es sólo el entrenamiento aeróbico el que puede mantener el buen estado de salud.

\section{PALABRAS CLAVE}

Entrenamiento de resistencia, diabetes, perfil glicémico.

\begin{abstract}
The present literature review arises from the need to review the evidence on the effect of resistance training on the glycemic profile. The search for information is carried out in the Ebsco, PubMed, Google Scholar and Researchgate databases, restricted to publications from the years 2011 to 2016. The following keywords were used: Resistance training and diabetes control, Resistance and glycemic control, Resistance Training and Health Control. 841 articles were found. Then, articles that did not meet the inclusion criteria of the research were excluded, and from those, 75 more were excluded because they did not manifest the effect of resistance training on glucose. Finally, only 6 research studies were selected and analyzed for this investigation. The results of this review show that RT methods are considered important components when the main objective is to maintain good health by positively intervening on the glycemic profile and by minimizing risk factors inherent to a poor diet and a sedentary lifestyle, and therefore demonstrating that it is not just aerobic training that can maintain good health.
\end{abstract}

\section{Key words}

Resistance training, diabetes, glycemic profile.

* Grupo de Estudos e Pesquisas em Respostas Neuromusculares - GEPREN, Universidade Federal de Lavras, Lavras, Brasil.

** Programa Magíster en Ciencias de la Motricidad Humana, Universidad Pedro de Valdivia, Chillán, Chile.

*** Departamento de Ciencias de la Actividad Física, Universidad de Los Lagos, campus Puerto Montt, Chile.

**** Laboratorio de Biociencias del Movimiento Humano (LABIMH). Universidad Federal de Rio de Janeiro, Brasil.

***** Facultad de Educación y Ciencias Sociales, Carrera Educación Física, Universidad Adventista de Chile. 


\section{INTRODUCCIÓN Y PLANTEAMIENTO DEL PROBLEMA}

La diabetes actualmente es considerada una de las mayores patologías a nivel mundial, se estima que, en todo el mundo 380 millones de personas padezcan esta dolencia (Quílez Llopis \& Reig García-Galbis, 2015), algunos estudios han demostrado que la diminución de la masa muscular en individuos con diabetes tipo 2 aumenta el riesgo de desarrollar intolerancia a la glucosa (Mahdirejei et al., 2014), siendo que ese tipo de diabetes es considerada la más prevalente dolencia crónica del siglo XXI (Daly et al., 2014).

La actividad física regular está asociada a una mayor longevidad y a una menor frecuencia de dolencias, siendo que, su práctica ayuda en el control glicémico, lo que es esencial para prevenir varias complicaciones relacionadas a la diabetes, como por ejemplo: la ceguera, diálisis, amputaciones y dolencias cardiovasculares que afectan la calidad de vida (Ishiguro et al., 2015; Little et al., 2011; Yardley et al., 2012). A finales del siglo pasado hubo un aumento explosivo de la expectativa de vida en todo el mundo, principalmente en los países desarrollados (Almeida, Silva, Santini, \& Dos-Reis, 2012; Paula, Cunha, \& Tufanin, 2014), y en la medida que el organismo va envejeciendo hay una alteración en el funcionamiento de varios sistemas orgánicos y también diminución de la masa muscular y como consecuencia pérdida de la fuerza muscular, diminución da función cardiorrespiratoria, dislipidemias y mayor resistencia a la insulina (Geirsdottir et al., 2015; Reis, Vitto, Vieira, \& Voltarelli, 2013). Cuando la absorción de la glucosa por el organismo es perjudicial y no hay un control glicémico efectivo, esto se traduce en hiperglicemia, que es considerada un factor de riesgo para complicaciones micro y macrovasculares como la retinopatía, nefropatía, neuropatía periférica, neuropatía autonómica y enfermedades cardiovasculares (De Souza et al., 2013, Pinheiro et al., 2013). Una de las formas de tratamiento y prevención de la hiperglicemia, es la práctica de ejercicios físicos, ya que el ejercicio aeróbico (EA) es prescrito con mayor frecuencia de los que los entrenamientos resistidos (ER) (Paula et al., 2014), o sea, ejercicios continuos de intensidad baja y moderada con un mínimo de 30 minutos de ejecución (Little et al., 2011). En los últimos años, el ER ha sido muy indicado para la mejora de la aptitud física y la calidad de vida de la población, pues este tipo de ejercicio es utilizado para el desarrollo de la fuerza, potencia, hipertrofia, resistencia muscular y también contribuye en el tratamiento de patologías crónicas no transmisibles, como la diabetes, hipertensión y la osteoporosis que se reflejan directamente en el estado de salud de las personas. Además, hay que señalar que la Asociación Americana de Diabetes (ADA) y el Colegio Americano de Medicina del Deporte (ACSM), recomiendan que el alumno con diabetes tipo 2 debe realizar ejercicio, para que pueda disminuir los niveles de glucosa en sangre y tener mayor seguridad antes de ingresar en un programa de entrenamiento (Francois \& Little, 2015). Sin embargo, es destacable que, los ejercicios más utilizados en la actualidad han sido los EA para la mejora en el perfil glicémico. Por lo tanto, el objetivo de esta investigación fue identificar los estudios que en la actualidad utilizaron los resultados de ER en el control glucémico en pacientes con diabetes tipo 2 .

\section{DESARROLLO}

A continuación se pretende dar a conocer abordajes teóricos y distintos tipos de intervenciones y los efectos del entrenamiento resistido en el perfil glicémico.

Actualmente el ER y el EA se tornaron una estrategia de entrenamiento bastante recomendada para personas con diabetes tipo 2 debido a sus efectos beneficiosos sobre la masa muscular, la glucemia y otros factores de riesgo que contribuyen para el desarrollo de la diabetes (Daly et al., 2014). En la investigación de Paula, Cunha, y Tufanin (2014), evaluaron los efectos del ER sobre los marcadores glucémicos, pero con una mayor utilización del EA que el ER. No obstante, lo que es evidente en la literatura es que el ER en conjunto con el EA, tiene la capacidad de disminuir la grasa corporal, especialmente la abdominal y visceral, así como la glucemia post prandial, o sea, tanto el ER como el EA son eficaces para la mejora 
de la aptitud física, siendo así, esta desarrolla un papel esencial en la promoción de la salud de sus practicantes (De Souza et al., 2013, Park, Park, Lim, \& Park, 2015, Roma et al., 2013, Shaw, Shaw, \& Brown, 2015, Slentz et al., 2011). En el estudio de Hamasaki et al., (2015), utilizando una muestra de pacientes obesos con diabetes tipo 2, los autores detectaron que el ER de baja intensidad con movimiento lento, junto con el EA al 50\% de la FC, fue capaz de disminuir la masa corporal $(-2.2 \mathrm{~kg})$, la masa grasa corporal $(-2.1 \mathrm{~kg})$, y el porcentaje de grasa corporal (-1.1\%), mejorando así el porcentaje de lipoproteínas de alta densidad (HDL) (+9.8\%) y bajando las lipoproteínas de baja densidad (LDL) $(-7.7 \%)$ y la hemoglobina glucosilada (HbA1c) (-16\%), este último indicador de bienestar de los niveles de glucosa en sangre y en el torrente sanguíneo. En otro estudio, los autores confirmaron que los individuos con diabetes mellitus tipo 2, presentaron una pequeña mejora de la glucemia $(-8.5 \%)$, HbA1c (-5\%), LDL (-20.6\%), después de un ER, EA y de flexibilidad (Vinetti et al., 2015).

En la investigación de Liao et al., (2015), con 120 sujetos divididos en dos grupos: Grupo Control (GC) y Grupo Entrenamiento Aeróbico (GA), en que el GA practicó el entrenamiento 5 veces por semana, siendo de $30 \mathrm{minu}$ tos por sesión, con una intensidad moderada durante 3 meses, en que se comparó el exceso de masa corporal, el índice de masa corporal (IMC), LDL, HDL, la HbA1c entre los grupos $y$, al final de tres meses, los autores encontraron mejorías en el grupo GA, donde el exceso de masa corporal disminuyó en un $40 \%$, el IMC un 6.5\%, el LDL en un (10\%), el colesterol HDL aumentó un (10\%) y la HbA1c disminuyó un (3.5\%). Esta investigación muestra que este tipo de ejercicio es capaz de provocar mejoras en las variables analizadas. Estudios anteriores como el de Boudou et al., (2000), mostraron una mayor reducción de la $\mathrm{HbAlc},(9.00 \%$ a $6.25 \%$ ), cuando fue comparado a la referida investigación, pero la intensidad y el tiempo del entrenamiento se mostraron dispares al realizado anteriormente (EA realizado a $75 \%$ de $\mathrm{VO}_{2}$ pico durante 8 semanas), el que argumenta las discrepancias entre las investigaciones. Justificando los hallazgos de Castaneda et al., (2002), donde encontraron una reducción de $8.7 \%$ a $7.6 \%$, en el ER realizado de 3 veces a la semana durante 16 semanas, siendo la intensidad del 60-80\% 1 RM 1-8 a semana y entre el $70-80 \%$ de $8-14^{\text {a }}$ semana, el que demuestra que tanto el tipo de estímulo (EA o ER) como la intensidad o el volumen pueden interferir en las respuestas glucémicos al ejercicio físico. En el estudio De Souza et al., (2013), realizado con 159 sujetos, donde compararon tres metodologías de entrenamiento, entrenamiento resistido (ER), entrenamiento aeróbico (EA) y entrenamiento combinado (EC), concluyeron que cuando compararon los tipos de entrenamiento, el ER fue lo que consiguió bajar más los niveles de la glucosa sanguínea de (198,7 $\mathrm{mg} / \mathrm{dl}$ a $179.5 \mathrm{mg} / \mathrm{dl}$ ), o sea, una disminución de 9.7\%. En el estudio realizado por Little et al., (2011) con un entrenamiento aeróbico de alta intensidad (High intensity Training - HIT) y de bajo volumen, donde se aplicó un protocolo de entrenamiento espaciado, que consistía en 10 conjuntos de estímulos de 60 segundos a $90 \%$ de la frecuencia cardíaca máxima intercalados con 60 segundos de descanso más de 2 semanas, constataron que el HIT obtuvo buenos resultados en la diminución de los niveles de glucosa sanguínea.

\section{METODOLOGÍA}

La muestra está compuesta por artículos de investigación relativos al ER de tipo estudio clínico aleatorizado, y extraídos de la base de datos EBSCO, PUBMED, GOOGLE ACADEMICO, RESEARCHGATE. La búsqueda y extracción de información fue realizada en Enero de 2016, y fueron almacenados en el gestor de referencias bibliográficas Endnote $\mathrm{X} 7.5$. Con respecto a las estrategias de búsqueda utilizadas en las bases de datos mencionadas, en primer lugar, los resultados se filtraron atendiendo a su objeto de análisis, considerando solo aquellos documentos que tienen como temática principal o incluyen en su muestra de estudio el ER. Para ello, se utilizaron las palabras clave, Resistance Training and control Diabetes, Resistance Training and control Glycemic, Resistance Training and health control. En segundo lugar, se incluyeron criterios de inclusión, publicados en idioma portugués, español o inglés, con fecha comprendida en el 
periodo 2011-2016, que estuvieran disponibles de manera íntegra y gratuita; se relacionaran precisamente con el tema en cuestión, que fueran realizados en personas adultas de ambos géneros, no dependientes de insulina y que no fueran deportistas. De esta manera, fueron excluidos los artículos que limitaban la interpretación de los datos o que no contaba con los criterios de inclusión ya mencionados. Por lo tanto, solamente seis (6) artículos cumplieron con estos requisitos. En la Tabla 1, son presentados los estudios de entrenamiento de fuerza aplicados en la mejora del perfil glicémico, con datos generales, características de la muestra, características de los entrenamientos de fuerza y los resultados obtenidos.

\section{Tabla 1}

Características de los estudios de entrenamiento de fuerza aplicados en la mejora del perfil glicémico.

\begin{tabular}{|c|c|c|c|}
\hline Título & $\begin{array}{c}\text { Características de la } \\
\text { muestra }\end{array}$ & $\begin{array}{c}\text { Características del entrenamiento } \\
\text { de fuerza }\end{array}$ & Resultados \\
\hline $\begin{array}{l}\text { Divide and con- } \\
\text { quer the ultidisci- } \\
\text { plinary approach } \\
\text { to achieving } \\
\text { significant long- } \\
\text { term weight loss } \\
\text { and improved } \\
\text { glycemic control } \\
\text { in obese patients } \\
\text { with type } 2 \text { diabe- } \\
\text { tes. Bishay, Omari, } \\
\text { Zang, Lih, y Kor- } \\
\text { mas (2013) }\end{array}$ & $\begin{array}{l}47 \text { sujetos sedentarios } \\
\text { evaluados, Divididos en } \\
45 \% \text { Hombres y } 55 \% \text { de } \\
\text { Mujeres. De ellos, un } \\
72 \% \text { era caucásicos, } 21 \% \\
\text { mediterránea, y el res- } \\
\text { to dividido entre origen } \\
\text { hispano, asiático, indio } \\
\text { (7\%). La edad promedio } \\
\text { fue de } 59,1 \pm 9,4 \text { años } \\
\text { (rango } 33-72 \text { años), y el } \\
\text { peso medio de } 104,7 \pm \\
19,9 \text { kg. Todos los sujetos } \\
\text { debían cumplir con estos } \\
\text { requisitos: } \\
\text { 1) IMC > } 30 \text { kg / m } 2 \text {. } \\
\text { 2) Padecer diabetes tipo } \\
\text { 2. Con ello, el grupo pre- } \\
\text { senta un alto riesgo de } \\
\text { cardiometabólico }\end{array}$ & $\begin{array}{l}\text { Sesiones de ejercicios incluyen una } \\
\text { combinación de resistencia (series } \\
3 \text {, repeticiones } 8 \text { a } 15 \mathrm{RMs} \text {, intensi- } \\
\text { dad y aeróbico ( } 60-80 \% \text { del máxi- } \\
\text { mo de respuesta de la frecuencia } \\
\text { cardíaca), una frecuencia semanal } \\
\text { 3x, y con duración de } 30 \text { meses. }\end{array}$ & $\begin{array}{l}\text { Reducción del peso }(7,9 \\
\pm 0,8 \%) \text {, IMC }(8.4 \pm \\
1.1 \%) \text {, circunferencia de } \\
\text { la cintura }(6,5 \pm 0,9 \%) \text {, } \\
\text { exceso de peso corporal } \\
(25,3 \pm 2,8 \%) \text {. Reduccio- } \\
\text { nes significativas en la } \\
\text { presión arterial sistólica } \\
(6,4 \pm 1,99 \%, P=0,015) \\
\text { y la presión arterial dias- } \\
\text { tólica }(6,6 \pm 2,0 \%, P= \\
0,008) .\end{array}$ \\
\hline $\begin{array}{l}\text { Effect of Circuit } \\
\text { Resistance trai- } \\
\text { ning on Glycemic } \\
\text { Control of Fema- } \\
\text { les with Diabetes } \\
\text { Type II. Shabani, } \\
\text { Nazari, Dalili, y } \\
\text { Rad (2015) }\end{array}$ & $\begin{array}{l}\text { Veinte mujeres con so- } \\
\text { brepeso y obesos con } \\
\text { diabetes tipo } 2 \text { fueron } \\
\text { seleccionados al azar en } \\
\text { dos grupos (ejercicio del } \\
\text { circuito de resistencia y } \\
\text { control). La edad media } \\
\text { en el grupo de ERC y } \\
\text { de control fueron } 50,2 \pm \\
4,89 \text { años y } 51,3 \pm 6,63 \\
\text { años, respectivamente. }\end{array}$ & $\begin{array}{l}\text { Sesiones de ER con la frecuencia } \\
\text { semanal } 3 x \text {, series } 2 \text { a } 3 \text {, repeticio- } \\
\text { nes } 8 \text { a } 12 \text {, intensidad } 50 \text { a } 65 \% \text { de } \\
1 \mathrm{RM} \text {. }\end{array}$ & $\begin{array}{l}\text { Los resultados mostraron } \\
\text { cambios significativos en } \\
\text { la hemoglobina glicosila- } \\
\text { da }(\mathrm{HbA} 1 \mathrm{c}-13,9 \%) \text { y la } \\
\text { grasa subcutánea }(-5 \%) \\
\text { se observaron en el gru- } \\
\text { po de ERC }(\mathrm{P}=0,04, \mathrm{p}= \\
0,002 \text {, respectivamente). } \\
\text { Además, los hallazgos in- } \\
\text { dicaron inferior HbA1c } \\
\text { en el grupo ER después } \\
\text { de la intervención, en } \\
\text { comparación con el gru- } \\
\text { po control }(\mathrm{P}=0,04) \text {. }\end{array}$ \\
\hline
\end{tabular}




\begin{tabular}{|c|c|c|c|}
\hline Título & $\begin{array}{l}\text { Características de la } \\
\text { muestra }\end{array}$ & $\begin{array}{c}\text { Características del entrenamiento } \\
\text { de fuerza }\end{array}$ & Resultados \\
\hline $\begin{array}{l}\text { Effects of aerobic } \\
\text { versus resistan- } \\
\text { ce training on } \\
\text { glycemic control } \\
\text { in men with type } \\
2 \text { diabetes. Moe, } \\
\text { Augestad, Asvold, } \\
\text { y Flanders (2011) }\end{array}$ & $\begin{array}{l}\text { Veintiséis hombres con } \\
\text { diabetes tipo } 2 \text {, fueron } \\
\text { seleccionados al azar en } \\
\text { dos grupos (ejercicio } \\
\text { aeróbico [13] y entre- } \\
\text { namiento de resistencia } \\
{[13] \text { ), que no requieran }} \\
\text { ningún otro medica- } \\
\text { mento antidiabético que } \\
\text { la metaformina. La edad } \\
\text { media en el grupo de ER } \\
57.77 \pm 7.82 \text { y el grupo de } \\
\text { EA } 56.23 \pm 8.29 \text { años. }\end{array}$ & $\begin{array}{l}\text { Sesiones de ejercicios incluyen } \\
\text { uno grupo entrenamiento de re- } \\
\text { sistencia (series } 3 \text {, repeticiones } 8 \\
\text { a } 15 \text {, intensidad } 60 \% \text { de } 1 \text { RM) y } \\
\text { otro de entrenamiento aeróbico } \\
\text { (Una semana se mantuvo el } 75 \% \\
\text { del VO2MÁX, y en un día más } \\
\text { cada semana, los participantes se } \\
\text { ejercitaron de forma intermitente } \\
\text { en cinco combates de } 2 \text { min a } 85 \% \\
\text { Vo2 máx. Los participantes se ejer- } \\
\text { citaron tres veces a la semana du- } \\
\text { rante } 12 \text { semanas. Cada sesión fue } \\
\text { supervisada y duró } 45 \text { minutos. }\end{array}$ & $\begin{array}{l}\text { En el presente } 12 \text { sema- } \\
\text { nas de ensayo clínico } \\
\text { aleatorizado, entrena- } \\
\text { miento aeróbico y de } \\
\text { resistencia llevaron a } \\
\text { modestas reducciones } \\
\text { de la HbA1c }(-5 \%) \text {, el } \\
\text { colesterol total }(-5 \%) \text { en } \\
\text { los hombres con diabetes } \\
\text { tipo } 2 \text {. }\end{array}$ \\
\hline $\begin{array}{l}\text { Combined aero- } \\
\text { bic and resistance } \\
\text { exercise training } \\
\text { decreases peri- } \\
\text { pheral but not } \\
\text { central artery wall } \\
\text { thickness in sub- } \\
\text { jects with type 2 } \\
\text { diabetes. Schreu- } \\
\text { der, Munckhof, } \\
\text { Poelkens, Hop- } \\
\text { man, and Thijssen } \\
\text { (2015) }\end{array}$ & $\begin{array}{l}\text { Veinte sedentarios (es de- } \\
\text { cir. No hacer ejercicio fí- } \\
\text { sico regular) los hombres } \\
\text { con diabetes tipo } 2(>2 \\
\text { años desde el diagnósti- } \\
\text { co, } 59 \pm 6 \text { años de edad) y } \\
10 \text { hombres sedentarios, } \\
\text { de la misma edad ( } 58 \pm 7 \\
\text { años) sin diabetes fueron } \\
\text { reclutados. }\end{array}$ & $\begin{array}{l}\text { Se realizaron } 8 \text { semanas de entre- } \\
\text { namiento, } 3 \text { sesiones por semana. } \\
\text { El entrenamiento consistió en } 5 \\
\text { minutos de calentamiento, segui- } \\
\text { do por un circuito ER, donde se } \\
\text { llevan a cabo tres series de } 12 \text { re- } \\
\text { peticiones con un minuto entre } \\
\text { series intercalan con el ejercicio } \\
\text { aeróbico ( } 70-75 \% \text { de reserva FC). }\end{array}$ & $\begin{array}{l}\text { En el presente } 8 \text { sema- } \\
\text { nas de entrenamiento, } \\
\text { ejecutado con } 3 \text { series } \\
\text { de } 12 \text { repeticiones con } \\
\text { un intervalo de un mi- } \\
\text { nuto entre ejercicios se } \\
\text { intercalan con el ejercicio } \\
\text { aeróbico ( } 70-75 \%) \text {, que } \\
\text { mostró un pequeño por- } \\
\text { centaje de mejoría de la } \\
\text { glucosa en } 7,8 \% \text {. }\end{array}$ \\
\hline $\begin{array}{l}\text { Resistance Tra- } \\
\text { ining Leads to } \\
\text { Clinically Mea- } \\
\text { ningful Improve- } \\
\text { ments in Control } \\
\text { of Glycemia and } \\
\text { Muscular Stren- } \\
\text { gth in Untrained } \\
\text { Middle-aged Pa- } \\
\text { tients with type } 2 \\
\text { Diabetes Mellitus. } \\
\text { Hameed, Manzar, } \\
\text { Raza, Shareef, and } \\
\text { Hussain (2012) }\end{array}$ & $\begin{array}{l}\text { Cuarenta y ocho sujetos, } \\
\text { se incluyeron si eran con } \\
\text { edades (edad } 44,7 \pm 4,2 \\
\text { años), tenían niveles de } \\
\text { HbA1c entre } 6,6 \% \text { y el } \\
9,9 \% \text { en el mes pasado, el } \\
\text { nivel de glucosa en plas- } \\
\text { ma en ayunas }>126 \mathrm{mg} \\
\text { / dl, fueron previamente } \\
\text { inactivos (definida como } \\
\text { ausencia el entrenamien- } \\
\text { to de fuerza y }<150 \text { mi- } \\
\text { nutos de caminar a paso } \\
\text { ligero / moderado ejer- } \\
\text { cicio por semana y }<60 \\
\text { minutos de ejercicio vi- } \\
\text { goroso por semana en los } \\
6 \text { meses anteriores), y si } \\
\text { eran diagnosticados con } \\
\text { diabetes tipo } 2 \text { durante } \\
\text { más de } 6 \text { meses. Vein- } \\
\text { ticuatro sujetos fueron } \\
\text { asignado a cada grupo } \\
\text { (ER y el grupo control). }\end{array}$ & $\begin{array}{l}\text { Cumplido el entrenamiento de } \\
\text { resistencia en una frecuencia de } \\
3 \text { veces por semana y realizada } 3 \\
\text { series de } 10 \text { repeticiones con un } \\
65 \% \text { de } 1 \text { RM con un intervalo de } \\
\text { descanso entre } 2 \text { y } 3 \text { min. En las } \\
\text { cuatro semanas siguientes se rea- } \\
\text { lizó el mismo protocolo, pero al } 70 \\
\% \text { de } 1 \mathrm{RM} \text {. }\end{array}$ & $\begin{array}{l}\text { Este estudio demostró } \\
\text { que el modelo de pro- } \\
\text { grama de ER aplicado } \\
\text { fue eficiente principal- } \\
\text { mente en el control de } \\
\text { la glucemia (aumento de } \\
\text { la acción de la proteína } \\
\text { GLUT4, transportador } \\
\text { de glucosa causando la } \\
\text { reducción de HbA1c que } \\
\text { puede conducir a la re- } \\
\text { ducción del riesgo de en- } \\
\text { fermedad cardiovascular } \\
\text { en pacientes con diabetes } \\
\text { tipo 2), y también en la } \\
\text { mejora de la fuerza mus- } \\
\text { cular en sujetos de me- } \\
\text { diana edad. }\end{array}$ \\
\hline $\begin{array}{l}\text { Insulin sensitivity } \\
\text { not modulated } 24 \\
\text { to } 78 \text { h after acute } \\
\text { resistance exercise } \\
\text { in type } 2 \text { diabetes } \\
\text { patients. Gordon, } \\
\text { Fraser, Bird, y } \\
\text { Benson (2013) }\end{array}$ & $\begin{array}{l}\text { Diez individuos inactivos } \\
\text { con diabetes tipo } 2(\mathrm{n}=6 \\
\text { hombres, mujeres } \mathrm{N}=4) \\
\text { con una edad media } \pm \text { de } \\
61,8 \pm 7,2 \text { años, la altura } \\
\text { de } 169,7 \pm 7,7 \mathrm{~cm} \text { y masa } \\
\text { corporal de } 86,8 \pm 13,4 \\
\text { kg y voluntarios. }\end{array}$ & $\begin{array}{l}\text { Cumplido el entrenamiento de } \\
\text { resistencia en } 3 \text { series de } 10 \text { repe- } \\
\text { ticiones con hasta el } 45 \%, 60 \% \text { y } \\
75 \% \text { de } 1 \mathrm{RM} \text { en una sola sesión. }\end{array}$ & $\begin{array}{l}\text { Este estudio no encon- } \\
\text { tró ninguna mejora en el } \\
\text { resultado de la insulina } \\
24,48 \text { y } 72 \text { horas des- } \\
\text { pués del ejercicio en alta } \\
\text { intensidad, cuando fue } \\
\text { realizado solamente una } \\
\text { sesión de ejercicios de } \\
\text { resistencia. }\end{array}$ \\
\hline
\end{tabular}




\section{ANÁLISIS DE LAS INTERVENCIONES}

En el estudio realizado por Shabani, Nazari, Dalili, y Hassanzadeh (2015), los autores encontraron que los resultados del programa de ER ha tenido bajo impacto en los factores de riesgo metabólicos en personas con diabetes tipo 2, destacando que el estudio se realizó en un circuito que contenía 6 ejercicios, donde se estipuló el total de 8 repeticiones a la intensidad del 40-50\% de 1RM, con un intervalo de 1-2 minutos entre las series durante las 3 primeras semanas. A partir de la cuarta semana en los mismos seis ejercicios se realizaron, 2-3 series de 10 a 12 repeticiones con un intervalo de 2 a 3 minutos en la intensidad del 50-65\% de 1RM. El estudio busco seguir las bases de prescripción de Garber et al., (2011), pero los resultados mostraron pocas mejorías, y pueden ser reportados por la baja intensidad y volumen adoptados en el trabajo, que hace que a las 8 semanas de entrenamiento las personas, todavía se encuentren en un período inicial de ER, no provocando mejoras significativas en la glucemia. Las investigaciones analizadas demuestran a través de sus resultados que el ER es capaz de interferir positivamente en los niveles de la glucosa sanguínea provocando mejoras en el cuadro de diabetes de las personas. Cuando se analizó el impacto del ER en el perfil glicémico, en la mayor parte de los artículos hubo consenso, solo en dos estudios nos presentan bajos resultados como el de Moe et al., (2011), donde realizaron una intervención de 12 semanas de entrenamiento aeróbico y de resistencia en el que llevaron a modestas reducciones de la HbAlc (-5\%), el colesterol total $(-5 \%)$ en los hombres con diabetes tipo 2, y el de Gordon et al. (2013), Este estudio no encontró ninguna mejora en el resultado de la insulina 24, 48 y 72 horas después del ejercicio en alta intensidad, cuando fue realizado solamente una sesión de ejercicios de resistencia. En cuanto a los otros 4 estudios se observan mejoras en el perfil glicémico. Por lo tanto, es necesario que las futuras investigaciones apliquen métodos avanzado de ER, con el objetivo de evaluar y analizar su impacto en el perfil glicémico de individuos adultos y comparar con los métodos de ER ya aplicados, procurando así dar un mejor respaldo en la elaboración y prescripción de este tipo de entrenamiento con foco en el perfil glicémico.

\section{CONCLUSIÓN}

Los resultados de la revisión, muestran que el ER se puede considerar como un componente importante en el objetivo de prevención del estado de salud, ya que minimiza los factores de riesgo inherente a la mala alimentación y la vida sedentaria.

\section{REFERENCIAS BIBLIOGRÁFICAS}

Almeida, J. B., Silva, W. V., Santini, E., \& dos Reis Filho, A. D. (2012). Aplicação de diferentes tipos de treinamento de força em idosas: estudo de casos. Revista Brasileira de Prescrição e Fisiologia do Exercício (RBPFEX), 6(31), 7.

Bishay, R. H., Omari, A., Zang, J., Lih, A., \& Kormas, N. (2013). Divide and conquer: The multidisciplinary approach to achieving significant long-term weight loss and improved glycemic control in obese patients with type 2 diabetes. $\mathrm{Cli}$ nical Diabetes, 31(1), 14-20.
Boudou, P., De Kerviler, E., Vexiau, P., Fiet, J., Cathelineau, G., \& Gautier, J. (2000). Effects of a single bout of exercise and exercise training on steroid levels in middle-aged type 2 diabetic men: relationship to abdominal adipose tissue distribution and metabolic status. Diabetes \& Metabolism 26(6):450457. http://europepmc.org/abstract/ med/11173715

Castaneda, C., Layne, J. E., Munoz-Orians, L., Gordon, P. L., Walsmith, J., Foldvari, M., . . Nelson, M. E. (2002). A ran- 
domized controlled trial of resistance exercise training to improve glycemic control in older adults with type 2 diabetes. Diabetes Care, 25(12), 23352341.

Daly, R. M., Miller, E. G., Dunstan, D. W., Kerr, D. A., Solah, V., Menzies, D., \& Nowson, C. A. (2014). The effects of progressive resistance training combined with a whey-protein drink and vitamin D supplementation on glycaemic control, body composition and cardiometabolic risk factors in older adults with type 2 diabetes: study protocol for a randomized controlled trial. Trials, 15, 431-431. doi: 10.1186/1745-6215-15-431

de Souza, F. P. L., da Silva Cruz, P. W., Neta, J. S. M. F., de Azevedo Tavares, M. C., França, J. A. L., \& Vancea, D. M. M. (2013). Effect of the intervention of different methods of training on postprandial blood glucose of type 2 diabetics. ConScientiae Saúde, 12(2), 227.

Francois, M. E., \& Little, J. P. (2015). Effectiveness and Safety of High-Intensity Interval Training in Patients With Type 2 Diabetes. Diabetes Spectrum, 28(1), 3944 36p. doi: 10.2337/diaspect.28.1.39

Garber, C. E., Blissmer, B., Deschenes, M. R., Franklin, B. A., Lamonte, M. J., Lee, I.-M., ... Swain, D. P. (2011). Quantity and Quality of Exercise for Developing and Maintaining Cardiorespiratory, Musculoskeletal, and Neuromotor Fitness in Apparently Healthy Adults: Guidance for Prescribing Exercise. $\mathrm{Me}$ dicine and Science in Sports and Exercise, 43(7), 1334-1359. doi: 10.1249/ $\underline{\text { MSS.0b013e } 318213 \mathrm{fefb}}$

Geirsdottir, O. G., Arnarson, A., Ramel, A., Briem, K., Jonsson, P. V., \& Thorsdottir, I. (2015). Muscular strength and physical function in elderly adults 6-18 months after a 12 -week resistance exercise program. Scandinavian journal of public health, 43(1), 76-82.
Gordon, B. A., Fraser, S. F., Bird, S. R., \& Benson, A. C. (2013). Insulin sensitivity not modulated 24 to $78 \mathrm{~h}$ after acute resistance exercise in type 2 diabetes patients. Diabetes, Obesity \& Metabolism, 15(5), 478-480. doi: 10.1111/ dom.12057

Hamasaki, H., Kawashima, Y., Tamada, Y., Furuta, M., Katsuyama, H., Sako, A., \& Yanai, H. (2015). Associations of Low-Intensity Resistance Training with Body Composition and Lipid Profile in Obese Patients with Type 2 Diabetes. PLoS One, 10(7), e0132959.

Hameed, U. A., Manzar, D., Raza, S., Shareef, M. Y., \& Hussain, M. E. (2012). Resistance training leads to clinically meaningful improvements in control of glycemia and muscular strength in untrained middle-aged patients with type 2 diabetes mellitus. North American journal of medical sciences, 4(8), 336.

Ishiguro, H., Kodama, S., Horikawa, C., Fujihara, K., Hirose, A. S., Hirasawa, R., . . . Sone, H. (2015). In Search of the Ideal Resistance Training Program to Improve Glycemic Control and its Indication for Patients with Type 2 Diabetes Mellitus: A Systematic Review and MetaAnalysis. Sports Medicine, 46(1), 67-77. doi: 10.1007/s40279-015-0379-7

Liao, H.-c., Zhong, S.-g., Li, P., Chen, W.-b., Cheng, C., Wang, Y.-g., . . . Xiao, C. (2015). Effects and mechanism of moderate aerobic exercise on impaired fasting glucose improvement. Lipids Health Dis, 14(1), 1.

Little, J. P., Gillen, J. B., Percival, M. E., Safdar, A., Tarnopolsky, M. A., Punthakee, Z., . . . Gibala, M. J. (2011). Low-volume high-intensity interval training reduces hyperglycemia and increases muscle mitochondrial capacity in patients with type 2 diabetes. Journal of Applied Physiology, 111(6), 1554-1560. 
Mahdirejei, H. A., Abadei, S. F. R., Seidi, A. A., Gorji, N. E., Kafshgari, H. R., Pour, M. E., ... Khayeri, M. (2014). Effects of an eight-week resistance training on plasma vaspin concentrations, metabolic parameters levels and physical fitness in patients with type 2 diabetes. Cell Journal (Yakhteh), 16(3), 367.

Moe, B., Augestad, L. B., Åsvold, B. O., \& Flanders, W. D. (2011). Effects of aerobic versus resistance training on glycaemic control in men with type 2 diabetes. European journal of sport science, 11(5), 365-374.

Park, J.-H., Park, H., Lim, S.-T., \& Park, J.-K. (2015). Effects of a 12-week healthylife exercise program on oxidized lowdensity lipoprotein cholesterol and carotid intima-media thickness in obese elderly women. Journal of physical therapy science, 27(5), 1435.

Paula, C. C., Cunha, R. M., \& Tufanin, A. T. (2014a). Análise do impacto do treinamento resistido no perfil lipídico de idosos. Revista brasileira de ciência e movimento, 22(1), 150-156.

Paula, C. C., Cunha, R. M., \& Tufanin, A. T. (2014b). Análise do impacto do treinamento resistido no perfil lipídico de idosos. DOI: http://dx.doi. org/10.18511/0103-1716/rbcm. v22n1p150-156

Pinheiro, CP., Oliveira, MDP., Faro, GBdA., Silva, EC., Rocha, EAAd., Barreto-Filho, JAS., . . . Sousa, ACS. (2013). Valor prognóstico da hiperglicemia de estresse na evolução intra-hospitalar na coronariopatia aguda. Arq Bras Cardiol, 100(2), 127-134.
Quílez Llopis, P., \& Reig García-Galbis, M. (2015). Control glucémico a través del ejercicio físico en pacientes con diabetes mellitus tipo 2; revisión sistemática. Glycemic Control Through Physical Exercise in type 2 Diabetes Systematic Review, 31(4), 1465-1472. doi: 10.3305/ $\underline{\text { nh.2015.31.4.7907 }}$

Reis Filho, A. D. d., Vitto, J. P. P., Vieira Junior, R. C., \& Voltarelli, F. A. (2013). Análise da glicemia capilar após 12 semanas de treinamento resistido em mulheres. Revista brasileira de ciência e movimento, 21(3), 150-156.

Roma, M. F. B., Busse, A. L., Betoni, R. A., Melo, A. C. d., Kong, J., Santarem, J. M., \& Jacob Filho, W. (2013). Efeitos das atividades físicas resistida e aeróbia em idosos em relação à aptidão física $\mathrm{e}$ à funcionalidade: ensaio clínico prospectivo. Einstein (São Paulo), 11(2), 153-157.

Schreuder, T., Munckhof, I., Poelkens, F., Hopman, M., \& Thijssen, D. (2015). Combined aerobic and resistance exercise training decreases peripheral but not central artery wall thickness in subjects with type 2 diabetes. Eur J Appl Physiol, 115(2), 317-326. doi: 10.1007/s00421014-3016-5

Shabani, R., Nazari, M., Dalili, S., \& Hassanzadeh Rad, A. (2015). Effect of Circuit Resistance Training on Glycemic Control of Females with Diabetes Type II. International Journal of Preventive Medicine, 6, 39-42. doi: 10.4103/2008$\underline{7802.154923}$

Shabani, R., Nazari, M., Dalili, S., \& Rad, A. H. (2015). Effect of Circuit Resistance Training on Glycemic Control of Females with Diabetes Type II. International Journal of Preventive Medicine, 6, 34. doi: 10.4103/2008-7802.154923 
Shaw, B. S., Shaw, I., \& Brown, G. A. (2015). Resistance exercise is medicine: Strength training in health promotion and rehabilitation. International Journal of Therapy and Rehabilitation, 22(8), 233.

Slentz, C. A., Bateman, L. A., Willis, L. H., Shields, A. T., Tanner, C. J., Piner, L. W., . . . Nelson, R. C. (2011). Effects of aerobic vs. resistance training on visceral and liver fat stores, liver enzymes, and insulin resistance by HOMA in overweight adults from STRRIDE AT/ RT. American Journal of PhysiologyEndocrinology and Metabolism, 301(5), E1033-E1039.

Vinetti, G., Mozzini, C., Desenzani, P., Boni, E., Bulla, L., Lorenzetti, I., . . Assanelli, D. (2015). Supervised exercise training reduces oxidative stress and cardiometabolic risk in adults with type 2 diabetes: a randomized controlled trial. Scientific reports, 5.

Yardley, J. E., Kenny, G. P., Perkins, B. A., Riddell, M. C., Malcolm, J., Boulay, P., ... Sigal, R. J. (2012). Effects of performing resistance exercise before versus after aerobic exercise on glycemia in type 1 diabetes. Diabetes Care, 35(4), 669-675. doi: 10.2337/dc11-1844

\section{Dirección para correspondencia}

Claudio Hernández-Mosqueira

Académico Departamento Ciencias de la Actividad Física, Universidad de Los Lagos, Puerto Montt, Chile.

Contacto:

claudio.hernandez@ulagos.cl

Recibido: 04-04-2017

Aceptado: 18-09-2017 